\title{
Effect of Aerobic Versus Anaerobic Exercise on Quality of Life in Stroke Patients
}

\author{
Mahmoud Y. Elzanaty' ${ }^{1}$, Mai M. Gamal', Gehan M. Ahmed ${ }^{3}$, Amira M. Abdel Rahman ${ }^{4}$, \\ Rania M. Tawfik ${ }^{5, *}$

\begin{abstract}
${ }^{1}$ Physical Therapy for Neuromuscular Disorders and its Surgery, Faculty of Physical Therapy, Cairo University and Deraya University, Egypt

${ }^{2}$ Physical Therapy for Neuromuscular Disorders and its Surgery, Faculty of Physical Therapy, Badr University, Cairo, Egypt ${ }^{3}$ Physical Therapy for Neuromuscular Disorders and its Surgery, Faculty of Physical Therapy, Cairo University, Cairo, Egypt ${ }^{4}$ Faculty of Medicine, Badr University, Cairo, Egypt

${ }^{5}$ Physical Therapy for Neuromuscular Disorders and its Surgery, Faculty of Physical Therapy, Cairo University, Cairo, Egypt
\end{abstract}

Received June 30, 2021; Revised August 16, 2021; Accepted September 2, 2021

\section{Cite This Paper in the following Citation Styles}

(a): [1] Mahmoud Y. Elzanaty, Mai M. Gamal, Gehan M. Ahmed, Amira M. Abdel Rahman, Rania M. Tawfik, "Effect of Aerobic Versus Anaerobic Exercise on Quality of Life in Stroke Patients," International Journal of Human Movement and Sports Sciences, Vol. 9, No. 6, pp. 1362 - 1370, 2021. DOI: 10.13189/saj.2021.090632.

(b): Mahmoud Y. Elzanaty, Mai M. Gamal, Gehan M. Ahmed, Amira M. Abdel Rahman, Rania M. Tawfik (2021). Effect of Aerobic Versus Anaerobic Exercise on Quality of Life in Stroke Patients. International Journal of Human Movement and Sports Sciences, 9(6), 1362 - 1370. DOI: 10.13189/saj.2021.090632.

Copyright $\bigcirc 2021$ by authors, all rights reserved. Authors agree that this article remains permanently open access under the terms of the Creative Commons Attribution License 4.0 International License

\begin{abstract}
Background: Stroke is one of the world's leading causes of death and long-term lack of ability. Objectives: This study aimed to test the effect of aerobic versus anaerobic exercise on stroke patients' quality of life. Methods: Thirty male patients with stroke enrolled in this study. The patients were divided into three groups at random: two research groups (GA) and (GB) and one control group (GC). A traditional physical-therapy program was provided for the control group $(\mathrm{GC})$ including: Passive Range of motion exercises for tightens muscles, Facilitation for weak muscles through: PNF technique, quick stretching. Besides traditional physical therapy, an aerobic exercise was performed by the patients in a study group (GA). Other than traditional physical treatment, patients received anaerobic training in the study group (GB). The session time for each group was 50-60 min; it is conducted three times a week for eight weeks. The Outcome Measures: The Arabic version of a short form dimension test (SF-36) used to assess the QOL items of the SF-36 were divided into eight different domains. All groups were evaluated before and after intervention with a QOL questionnaire (SF36). Results: There was a statistical meaningful increase in physical functioning, (vitality) energy/fatigue, emotional well-being, social functioning, pain and general-health in study groups (GA), (GB)
\end{abstract}

compared with that of control group (GC) after therapy. However, there was no significant difference between groups in role limitations due to physical health and role limitations due to emotional problems. There was no meaningful difference in the quality of life items within groups (GA), (GB) after therapy. Conclusion: Eight weeks of training revealed non-significant difference between aerobic and anaerobic groups. However, either aerobic or anaerobic training has a beneficial effect on quality of life in stroke patients.

Keywords Stroke, Aerobic Training, Anaerobic Training, Quality of Life

\section{Introduction}

Stroke is among the major causes of death and long-term disability globally [1]. It has pernicious long-term impacts, resulting in a negative spiral of declines in cardiovascular fitness, mobility, and functional autonomy, as well as sedentary behaviour [2]. Walking, balancing, and upper limb functions are all influenced by the common impairments associated with strokes, such as loss of strength, feeling, and coordination. This can have a 
measurable influence on a one's health, safety, also overall quality of life [3].

Regular aerobics is suggested by the American Heart Institutes part of stroke prevention and treatment [4]. Aerobics (AE) is outlined as "designed or constructed repetitive physical activity for long durations and at sufficient intensity to improve physical fitness." It is proposed that AE be picked up on most days of the week at intensity that gradually increased for at least eight weeks [5]. Aerobics (AE) incorporated into post-stroke rehabilitation programs greatly improves function and prevent repeated strokes. In best practice and clinical standards, it's referred to as part of comprehensive stroke recovery [6].

Another modality referred to as anaerobic exercise (also referred to as strength exercise or resistance exercise). The American College of Physicians (ACSM): stated it "intense, short-duration physical activity powered by energy sources inside the muscle" [7]. Strength training, in contrast to aerobic exercise, is still an understudied exercise modality for stroke patients; strength training can enhance one's functionality, psychological and social elements, and therefore life-quality in stroke patients [8].

Stroke survivors can undergo neurological disorders like cognitive, communication, physical, and emotional impairment, resulting in either major or minor disability and limiting their ability to overcome premorbid functioning in number of lives domains, including physical, psychological, social activities [9]. Survivor's quality of life may be negatively impacted for many years after the event [10]. The World Health Organization (WHO) defines quality of life as the "perception of the person's life position within the context of the culture and value systems in which he or she lives and with respect to his or her goals" [11].

After a successful stroke recovery, patients must be able to return to their previous degree of performance of life and community involvement. There were extensively complaints of the lack of opportunities and research on long-terms impacts of stroke [12].

Previous researches have been done on either aerobic or anaerobic exercise's contribution to QOL in stroke patients $[13,14]$, so that this study's contribution is primarily in comparing the difference between the effect of these exercise types on stroke patients' quality of life.

\section{Methodology}

\section{Patients:}

The study was conducted on thirty male hemi-paretic patients following a stroke in the domain of the carotid artery. Based on a comprehensive clinical evaluation by a neurologist and radiological investigations including computed axial tomography and/or brain magnetic resonance imaging, the patients were diagnosed with a stroke within carotid system domain. The patients were picked from the Outpatient-Clinics of Cairo University's Faculty of Physical-Therapy. Patients were assigned at random (by sealed envelopes) into three equal groups, the two study groups (GA) and (GB) and the control group (GC).

\section{Study-design:}

This was a randomized controlled study, pre-test post-test design study. In this research design, before the procedure, the dependent variable's effect on groups was observable (pre-test). Later, the experimental and control groups were treated, and after that, the dependent variable was observed in the groups to see whether the manipulation of an independent variable had any power on the dependent variable (post-test).

\section{The patients were chosen under the following criteria:}

1. The Patient's age ranged from 50- 65 years

2. The duration of the illness was not less than six months and not more than two years.

3. Blood pressure of patients was examined for safety monitoring with resting systolic blood-pressure (SBP) $<150 \mathrm{~mm}-\mathrm{Hg}$ and/or resting diastolic BP (DBP) $<90$ $\mathrm{mm}-\mathrm{Hg}$.

4. Based on the modified Ashworth scale, muscle tone in the affected upper and lower limbs ranged from 1 to $1+$.

5. Depending on a manual muscle examination, Muscle strength in the upper and lower limbs affected is not less than grade three.

6. Motor recovery for both upper and lower limbs stage IV, $\mathrm{V}$ according to Brunnstrom motor recovery stages.

7. Body Mass Index (normally weighting (18.5-25) and for overweighting (25-30) $\mathrm{kg} / \mathrm{m} 2)$.

8. Patients with adequate cognitive abilities (Mini-Mental Scale >24) to understand and obey instructions.

\section{The present study removes patients with the following conditions:}

1. Any neurological conditions that have an adverse influence on quality of life except for stroke as (e.g., multiply-sclerosis, peripheral neuropathy, Parkinsonism).

2. Musculoskeletal problems such as extreme arthritis and fracture.

3. Cardiovascular or pulmonary conditions (unsettled angina, current myocardial infarction during the last three months, congestive heart failure, severe heart valve dysfunction).

4. All patients suffered from uncontrolled diabetes mellitus and uncontrolled hypertension.

5. Language or communication barrier that prevents the completion of questionnaires. 
6. Visual and auditory problems.

A verbal explanation about the important justification and main points of achievement of the study was explained to every patient.

\section{The procedures of the current study were divided into two main categories:}

Measurement procedures:

a) Initial evaluation procedures (initial phase)

- $\quad$ Each patient was medically tested to rule out all of the previously described abnormal medical issues.

- $\quad$ Each patient's medical history was documented in a questionnaire that included details such as name, age, BMI, and a determination of any functional, social, or psychological issues.

- For each patient in each category, the object of the assessment procedures was described in stages.

b) Technical measurements phases

The patients were assessed before and after the study using:

\section{Stroke quality of life assessment scale:}

The Arabic version of a short form dimension test (SF-36) used to assess stroke patient's life quality (QOL),

\section{Items of measure:}

All groups were evaluated before and after intervention with a QOL questionnaire (SF36). Items of the SF-36 were classified into eight different domains (Physical Functioning, Role Limitations due to Physical-Health, Role Limitations due to Emotional-Problems, (vitality) Energy/Fatigue,Emotional well-being, Social-Functioning, Bodily Pain and General-Health). Each patient was allowed to be familiar with the scale before starting the recording. To start the evaluation process, the patients were seated in a comfortable relaxed position; the patients were asked to respond to questions about their activities during the previous four weeks. Subscale items were added together to create a total score for each subscale or dimension.

\section{Weight and height scale:}

Before treatment, each patient's height and weight were calculated to determine their body mass index (BMI) using the following equation: $\mathrm{BMI}=$ weight $(\mathrm{Kg}) /$ height $(\mathrm{m} 2)$.

\section{Blood pressure monitoring by (Aneroid Sphygmomanometers):}

Aneroid sphygmomanometers were used to obtain a BP reading [15].

\section{Pulse-oximetry for (SPO2):}

At baseline and during clinical procedures, were widely used to noninvasively assess arterial oxyhemoglobin saturation $(\mathrm{SpO} 2)$ and heart rate $(\mathrm{HR})$ [16]. The use of a pulse oximeter will aid in achieving an exercise intensity that is within the recommended target heart rate range.

\section{Target heart rate:}

The minimum number of heartbeats in a given period of time required to achieve the level of exertion required for cardiovascular health, as determined by a person's age, gender, and physical fitness. Readings preferably were taken while the patient was seated comfortably in a chair. The patients were tested in a room with a comfortable degree of temperature.

\section{For monitoring resting heart rate:}

Taking pulse from the radial artery then count pulses for one minute [17].

- Maximum Heart Rate Formula: HRmax (pred) = 206.9 - (0.67 x age) [18].

- Calculate one's reserve heart-rate (HRR) by subtracting the resting rate of heart from the maximum heart rate. HRR is multiplied by 0.64 . (64 percent). Multiply this number by 0.76 and add the resting heart rate 0.76 (76 percent). To this, add your resting heart rate.

Moderate intensity aerobic exercise is described by The American College of Physicians (ACSM) as when person's rate of heart reaches 64-76 percent of their age-predicted peak heart rate [17].

\section{Therapeutic Procedures:}

Therapeutic intervention for the study was started at the same time for all groups, the session time for each group was 50-60 min divided as the following:

\section{Control group (GC):}

Patients in this group received Warm up (simple stretching exercise for all muscle groups) for 3-5 min: traditional physical training for (40-45 $\mathrm{min})$ three/times per week for eight weeks (in total 24 sessions), patients allowed to rest if necessary, Cool down (simple stretching exercise for all muscle groups) for (3-5 min).

\section{Traditional physical training program:}

- Passive Range of motion exercises for tightening muscles of upper and lower limbs 
- Facilitation for weak muscles through:

-Proprioceptive Neuromuscular Facilitation technique for affected upper and lower limbs. Patients allowed to rest if necessary

-Quick stretching for upper and lower limbs

\section{Study group (GA):}

The patients in this group received Warm up (simple stretching exercises for all muscle groups) for 3-5 min, the traditional physical training program for $(25-30 \mathrm{~min})$ in addition to aerobic exercise for (20-25 min) three/times per week for eight-weeks (in total 24 sessions); patients asked to rest if need, Cool down (simple stretching exercise for all muscle groups) for (3-5 $\mathrm{min})$.

\section{Aerobic training intervention:}

- Marching in place

- Overground walking (walking Exercise bouts)

\section{Study group (GB):}

The patients in this group received Warm up (simple stretching exercises for all muscle groups) for 3-5 min, the traditional physical training program for $(25-30 \mathrm{~min})$ in addition to anaerobic exercise for (20-25 $\mathrm{min})$ three/times per week for eight-weeks (in total 24 sessions); patients asked to rest if need, Cool down for (simple stretching exercise for all muscle groups) for (3-5 min).

\section{Anaerobic training intervention:}

Squat, Push-ups on Wall in Standing, Bridge Endurance Exercise, Sit-to-Stand, Toe Raises, Heel Raises.

\section{Data Analysis}

ANOVA-test was used to conduct the comparison of the mean age, BMI and length of illness between the three groups. Chi squared test was used to compare the affected side distribution between all groups. Kruskal-Wallis test was conducted for comparing the median values of quality-of-life items between the three groups and was followed by Mann. - Whitney $U$ test to indicate the significance difference between each two groups. Wilcoxon-Signed Ranks test was used to compare pre and post treatment median values of quality of life items in each group. The significance level for all statistical analyses was picked at $\mathrm{p} 0.05$. All statistical analysis was carried out using the statistical package for social studies (SPSS) version 25 for windows (IBM SPSS, Chicago, IL, USA).

\section{Results}

\section{Subjects characteristic:}

The subject characteristics of the groups GA, GB, and GC were shown in Table (1). There was no significant difference in age, BMI, duration of illness, or distribution of the affected side between groups ( $p>0.05$ ).

Table 1. Basic characteristics of the participants

\begin{tabular}{|c|c|c|c|c|}
\hline & Group. GA & Group. GB & Group. GC & P-value. \\
\hline Age., mean $\pm(S D)$, years & $58.8 \pm 5.45$ & $60.4 \pm 5.01$ & $61 \pm 3.88$ & 0.58 \\
\hline BMI, mean $\pm(\mathrm{SD}), \mathrm{kg} / \mathrm{m}^{2}$ & $25.84 \pm 2.5$ & $24.62 \pm 1.46$ & $25.58 \pm 2.13$ & 0.39 \\
\hline $\begin{array}{l}\text { Duration. of illness, mean } \pm(\mathrm{SD}) \text {, } \\
\text { month }\end{array}$ & $16.8 \pm 6.32$ & $17.5 \pm 5.6$ & $16.9 \pm 6.62$ & 0.96 \\
\hline \multicolumn{5}{|l|}{ Affected side, n (\%) } \\
\hline Dominant side & $6(60 \%)$ & $4(40 \%)$ & $5(50 \%)$ & \multirow{2}{*}{0.67} \\
\hline Non dominant side & $4(40 \%)$ & $6(60 \%)$ & $5(50 \%)$ & \\
\hline
\end{tabular}

*SD, *standard-deviation; p-value, level of significance 


\section{Effect of treatment on quality of life:}

\section{Within Group-comparisons}

Within group comparison showed a significant improvement in Physical functioning, (vitality) energy/fatigue, Emotional well-being, Social-functioning, Pain and General-health after treatment relative to before treatment in group GA, GB and GC ( $<$ < 0.05). However, there was no significant change in each of role-limitations due to physical health and role-limitations due to emotional problems between pre and post treatment in the three groups $(\mathrm{p}>0.05$.) (Table 2,3$)$.

\section{Comparison between groups}

Comparison between groups pre-treatment showed a non-significant change in all items $(p>0.05)$. There was a significant improvement in physical-functioning, energy/fatigue, emotional-well-being, social-functioning, pain and general health of group GA and GB compared with that of group GC after treatment $(p<0.05)$. There was no significant difference in role-restrictions due to physical-health and role-restrictions due to emotional problems between groups after treatment $(p>0.05)$. There was non-significant difference in quality-of-life items between group GA and GB after treatment ( $p>0.05$.). (Table 2,3).

Table 2. Median values of physical functioning, role limitations due to physical health, role limitations due to emotional problems, and energy/fatigue in groups GA, GB, and GC pre- and post-treatment

\begin{tabular}{|c|c|c|c|c|c|c|}
\hline & $\begin{array}{l}\text { Group. } \\
\text { GA }\end{array}$ & $\begin{array}{l}\text { Group. } \\
\text { GB }\end{array}$ & $\begin{array}{l}\text { Group. } \\
\text { GC }\end{array}$ & \multicolumn{3}{|c|}{ P-value. } \\
\hline & Median (IQR) & Median. (IQR) & Median. (IQR) & $\begin{array}{c}\text { GA vs. } \\
\text { GB }\end{array}$ & $\begin{array}{c}\text { GA vs. } \\
\text { GC }\end{array}$ & $\begin{array}{l}\text { GB vs. } \\
\text { GC }\end{array}$ \\
\hline \multicolumn{7}{|l|}{ Physical functioning } \\
\hline Pre-treatment & $47.5(61.25-31.25)$ & $40(56.25-23.75)$ & $40(51.25-20)$ & 0.49 & 0.22 & 0.64 \\
\hline \multirow[t]{2}{*}{ Post-treatment } & $75(81.25-68.75)$ & $70(76.25-26.5)$ & $52.5(66.25-36.25)$ & 0.28 & 0.002 & 0.02 \\
\hline & $p=0.005$ & $p=0.007$ & $p=0.01$ & & & \\
\hline \multicolumn{7}{|c|}{$\begin{array}{c}\text { Role-limitations due to } \\
\text { physical health }\end{array}$} \\
\hline Pre-treatment & $\mathbf{0}(\mathbf{0 - 0})$ & $\mathbf{0}(\mathbf{0 - 0})$ & $\mathbf{0}(6.25-\mathbf{0})$ & 1 & 0.54 & 0.54 \\
\hline \multirow[t]{2}{*}{ Post-treatment } & $\mathbf{0}(6.25-0)$ & $\mathbf{0}(\mathbf{0 - 0})$ & $\mathbf{0}(6.25-0)$ & 0.54 & 1 & 0.54 \\
\hline & $p=0.31$ & $p=1$ & $p=1$ & & & \\
\hline \multicolumn{7}{|c|}{$\begin{array}{c}\text { Role limitations due to } \\
\text { emotional problems }\end{array}$} \\
\hline Pre-treatment & $\mathbf{0}(8.33-\mathbf{0})$ & $\mathbf{0}(8.33-\mathbf{0})$ & $\mathbf{0}(0-\mathbf{0})$ & 0.91 & 0.14 & 0.14 \\
\hline \multirow[t]{2}{*}{ Post-treatment } & $\mathbf{0}(33.33-\mathbf{0})$ & $\mathbf{0}(8.33-0)$ & $\mathbf{0}(0-\mathbf{0})$ & 0.72 & 0.06 & 0.14 \\
\hline & $p=0.31$ & $p=1$ & $p=1$ & & & \\
\hline \multicolumn{7}{|l|}{ Energy/fatigue } \\
\hline Pre-treatment & $35(51.25-35)$ & $32.5(41.25-27.5)$ & $35(45-33.75)$ & 0.24 & 0.44 & 0.43 \\
\hline \multirow[t]{2}{*}{ Post-treatment } & $60(70-48.75)$ & $55(65-48.75)$ & $45(51.25-35)$ & 0.42 & 0.007 & 0.01 \\
\hline & $p=0.007$ & $p=0.004$ & $p=0.01$ & & & \\
\hline
\end{tabular}

IQR, Interquartile range; p-value, Level of significance 
Table 3. Median values of emotional wellbeing, social functioning, pain, and general health in groups GA, GB, and GC pre- and post- treatment

\begin{tabular}{|c|c|c|c|c|c|c|}
\hline & Group* GA & Group. GB & Group- GC & \multicolumn{3}{|c|}{ P-value. } \\
\hline & Median. (IQR.) & Mediann (IQR.) & Mediana (IQR.) & $\begin{array}{c}\text { GA vs } \\
\text { GB } \\
\end{array}$ & $\begin{array}{c}\text { GA vs } \\
\text { GC }\end{array}$ & $\begin{array}{c}\text { GBvs } \\
\text { GC }\end{array}$ \\
\hline \multicolumn{7}{|c|}{ Emotional well-being } \\
\hline Pre treatment & $46(56-25)$ & $40(57-16)$ & $40(48-32)$ & 0.62 & 0.51 & 0.84 \\
\hline \multirow[t]{2}{*}{ Post treatment } & $68(68-56)$ & $60(73-56)$ & $50(60-45)$ & 0.64 & 0.01 & 0.03 \\
\hline & $p=0.01$ & $p=0.005$ & $p=0.01$ & & & \\
\hline \multicolumn{7}{|l|}{ Social-functioning } \\
\hline Pre treatment & $43.75(50-37.5)$ & $\mathbf{5 0}(\mathbf{5 0}-25)$ & $43.75(53.12-25)$ & 0.96 & 1 & 0.84 \\
\hline \multirow[t]{2}{*}{ Post treatment } & $75(75-71.87)$ & $75(78.12-68.75)$ & $\mathbf{5 0}(75-50)$ & 0.92 & 0.006 & 0.03 \\
\hline & $p=0.004$ & $p=0.006$ & $p=0.01$ & & & \\
\hline \multicolumn{7}{|l|}{ Pain } \\
\hline Pre treatment & $72.5(87.5-64.37)$ & $65.25(80-65)$ & $45(66-32.5)$ & 0.94 & 0.9 & 0.9 \\
\hline \multirow[t]{2}{*}{ Post treatment } & $45(63.75-21.5)$ & $42.5(55.62-30)$ & $43.75(47.5-32.5)$ & 0.44 & 0.003 & 0.03 \\
\hline & $p=0.005$ & $p=0.005$ & $p=0.02$ & & & \\
\hline \multicolumn{7}{|l|}{ General-health } \\
\hline Pre-treatment & $37.5(45-30)$ & $32.5(41.25-25)$ & $30(41.25 .-28.75)$ & 0.35 & 0.24 & 0.96 \\
\hline \multirow[t]{2}{*}{ Post-treatment } & $\mathbf{5 5}(57.5-48.75)$ & $\mathbf{5 2 . 5}(56.25-\mathbf{4 3 . 7 5 )}$ & $35(46.25-35)$ & 0.43 & 0.001 & 0.009 \\
\hline & $p=0.005$ & $p=0.005$ & $p=0.005$ & & & \\
\hline
\end{tabular}

IQR, Interquartile range; p-value, Level of significance

\section{Discussion}

The aim of this research was to see how aerobic versus anaerobic exercise affected stroke patients' quality of life. This research included thirty male hemi-paretic patients who had suffered a stroke in the carotid artery domain. The patients were divided into three groups at random: two research groups: (GA) \& (GB) \& one control group (group GC). In the control group (GC) patients received the traditional physical therapy program. Besides traditional physical therapy, patients provided aerobic exercise in the study group (GA). Other than traditional physical therapy, patients provided anaerobic training in the study group (GB). The session time for each group was $50-60 \mathrm{~min}$; it is conducted three times a week for eight weeks.

Findings of this study showed a substantial change in group GA and group GB compared to group GC post treatment in the items of physical/functioning, (vitality) energy/fatigue, emotional-wellbeing, social functioning, pain and general-health of the SF-36 except for role constraints due to physical health and role/restrictions due to emotional problems where there was no significant difference .within groups pre and post treatment and they were the most affected domains in all groups. In addition, after therapy the current study revealed no meaningful difference in quality of life between groups GA and GB.

Schroeder et al. [19] supported the current study's findings by observing training-induced adaptations seen following aerobic and anaerobic (resistance training), as aerobic exercise led to a substantial improvement in cardiorespiratory fitness, while resistance training contributed to a significant improvement in muscular strength.

Furthermore, our findings are consistent with those of Santos et al. [20], who found that stroke affects the quality of life of elderly patients, with role restrictions due to physical health and role constraints due to emotional problems being the most affected domains.

Furthermore, Katz-Leurer et al.[21] confirmed that moderate-intensity aerobic exercise (64-76 percent of age-predicted peak heart rate) done for 20-25 minutes three days per week for eight weeks improves aerobic activity and walking endurance in stroke patients. Short exercise bouts can be offered to those with low exercise tolerance. (e.g., 2 minutes) with interspersed rest periods at the start

Similarly, Taheri et al.[22] found that daily walking and slow running enhances life quality both physically and psychologically, making it a healthy and low-cost technique for health professionals to use. Walking has a beneficial impact on physical health, stress management, and weight control, which may explain the findings.

Moreover, our finding is in line with Gordon et al.[13] who compared a group that received three days per week aerobic exercise plus classical physical therapy to a group that received only classical physical therapy for twelve weeks, and they used the short form-36 to assess quality of life (SF-36). The physical health component showed a significant difference between pre- and post-treatment 
scores and the patients felt more energetic and emotionally better after adding aerobic exercise to their regimen.

The aim of other researches was to see how anaerobic/resistance training affected the quality of life of stroke survivors. Strength training increases muscle strength in stroke patients by more than $75 \%$ from baseline, according to many reports [23, 24, 25].

Veldema et al.[26] confirmed that resistance training exceeds all other therapies in terms of supporting muscle power and motor function in either the involved and non-involved hemi-body. Furthermore, the selection of resistance training protocol is critical. High intensity training, eccentric training, and eccentric + concentric training are superior to low intensity training, concentric training, and isometric training in terms of efficiency. Furthermore, paretic muscle atrophy is strongly linked to decreased fitness levels.

Also our findings are supported by Aidar et al. [14] who explained that twelve weeks of anaerobic training resulted in a significant improvement in life quality indicators. Training in general, and anaerobic/strength training in particular, has been promoted as a means of improving functionality in stroke patients.

Moreover, Giuliano et al.[27] and Pedersen et al.[28] demonstrated in many cohorts that resistance training positively enhance independence and one's live quality.

Barrett et al.[29], in contrast, found non meaningful impact on the life quality for aerobic and anaerobic exercises. This owed to different methodologies and training protocols which could be to blame for these convergent results.

The possible limitation of our study is the small sample size, although small, was believed to be sufficient due to the experimental design used and the statistics used to draw some conclusions. Also, the short duration of the intervention may limit the study results. However, the study may help in the future research toward better results regarding the quality of life in stroke patients.

\section{Conclusions}

Eight weeks of training revealed non-significant difference between aerobic and anaerobic groups. However, either aerobic or anaerobic training has a beneficial effect on quality of life in stroke patients. Therefore, the present results emphasize that the addition of aerobic and /or anaerobic exercises to the traditional physical therapy program can be used as an effective method for improving stroke patient's life quality. However, further research is recommended to investigate the impact of longer durations of such intervention.

\section{Author Contributions}

M.Y.E and R.M.T,; Formal analysis, G.M.A,;
Investigation, M.M.G,; Methodology-writing-original draft, A.M.A and G.M.A,; Validation-writing-review\& editing, All authors have read and agreed to the published version of the manuscript.

\section{Funding}

This work was no funded.

\section{Institutional Review Board Statement}

This study was conducted according to the guidelines of the Declaration of Helsinki and approved by the Faculty of Physical Therapy Ethical Committee, Cairo University, Egypt (P.T.REC/012/002936).

\section{Informed Consent Statement}

Informed consent was obtained from all subjects involved in the study.

\section{Data Availability Statement}

The data presented in this study are available on request from the corresponding author.

\section{Conflicts of Interest}

The authors declare no conflict of interest.

\section{REFERENCES}

[1] Roth, G. A., Abate, D., Abate, K. H., Abay, S. M., Abbafati, C., Abbasi, N., ... \& Borschmann, R." Global, regional, and national age-sex-specific mortality for 282 causes of death in 195 countries and territories, 1980-2017: a systematic analysis for the Global Burden of Disease Study 2017," The Lancet, vol.392, no.10159, pp.1736-1788, 2018. DOI: 10.1016/S0140-6736(18)32203-7.

[2] Barrett, M., Snow, J. C., Kirkland, M. C., Kelly, L. P., Gehue, M., Downer, M. B., ... \& Ploughman, M."Excessive sedentary time during in-patient stroke rehabilitation," Topics in stroke rehabilitation,vol. 25, no.5, pp.366-374, 2018. DOI: $10.1080 / 10749357.2018 .1458461$

[3] Bower, K. J., Louie, J., Landesrocha, Y., Seedy, P., Gorelik, A., Bernhardt, J. "Clinical feasibility of interactive motion-controlled games for stroke rehabilitation," Journal of neuroengineering and rehabilitation, vol. 12, no.1, pp.63, 2015. DOI: $10.1186 / \mathrm{s} 12984-015-0057-\mathrm{X}$

[4] Winstein, C. J., Stein, J., Arena, R., Bates, B. Cherney, L. R., Cramer, S. C., ... \& Zorowitz, R. D. "Guidelines for adult 
stroke rehabilitation and recovery: a guideline for healthcare professionals from the American Heart Association /American Stroke Association," Stroke, vol. 47, no.6, pp.e98-e169, 2016. DOI: 10.1161/STR.00000000000 00098.

[5] MacKay-Lyons, M., Billinger, S. A., Eng, J. J., Dromerick, A., Giacomantonio, N., Hafer-Macko, C., ... \& Unsworth, K. "Aerobic exercise recommendations to optimize best practices in care after stroke: AEROBICS 2019 update," Physical therapy, vol. 100, no. 1, pp.149-156, 2020. DOI: 10.1093/ptj/pzz153.

[6] Gezer, H., Karaahmet, O. Z., Gurcay, E., Dulgeroglu, D., \& Cakci, A. "The effect of aerobic exercise on stroke rehabilitation," Irish Journal of Medical Science, vol. 188, no.2, pp. 469-473, 2019. DOI: 10.1007/s11845-018-18484.

[7] Pescattelo LS. \& American College of Sports Medicine (Eds.)."ACSM's guidelines for exercise testing and prescription," $9^{\text {th }} \mathrm{ed}$, Lippincott Williams \& Wilkins., 2014, pp. 91-114.

[8] Han, P., Zhang, W., Kang, L., Ma, Y., Fu, L., Jia, L., ... \& Guo, Q. " Clinical evidence of exercise benefits for stroke. Exercise for cardiovascular disease prevention and treatment," Adv Exp Med Biol, vol. 1000, pp.131-151,2017.DOI:10.1007/978-981-10-4304-8_9.

[9] Ellis, C., Grubaugh, A. L., \& Egede, L. E. "The association between major depression, health behaviors, and quality of life in adults with stroke," International journal of stroke, vol. 7, no. 7, pp. 36- 543, 2012. DOI:10.1111/j.1747-4949.2011.00708.x.

[10] Pinkney, J. A., Gayle, F., Mitchell-Fearon, K., \& Mullings, J. "Health-related quality of life in stroke survivors at the university hospital of the west indies," Journal of Neurology Research, vol. 7, no. 3, pp.46-58, 2017. DOI: $10.14740 /$ jnr $422 \mathrm{w}$

[11] Ansari, S. H., Baghersalimi, A., Azarkeivan, A., Nojomi, M., \& Rad, A. H. "Quality of life in patients with thalassemia major," Iranian journal of pediatric hematology and oncology, vol. 4, no. 2, pp.57-63, 2014.

[12] Obembe, A. O., \& Eng, J. J. "Rehabilitation interventions for improving social participation after stroke: a systematic review and meta-analysis," Neurorehabilitation and neural repair, vol.30, no.4, pp.384-392, 2016 DOI: $10.1177 / 1545968315597072$

[13] Gordon, C. D., Wilks, R., \& McCaw-Binns, A. "Effect of aerobic exercise (walking) training on functional status and health-related quality of life in chronic stroke survivors: randomized controlled trial," Stroke, vol. 44, no.4, pp.1179-1181,2013.DOI:10.1161/STROKEAHA.111.0006 42

[14] Aidar, F. J., de Oliveira, R. J., Silva, A. J., de Matos, D. G., Mazini Filho, M. L., Hickner, R. C., \& Machado Reis, V. "The influence of resistance exercise training on the levels of anxiety in ischemic stroke," Stroke research and treatment, vol. 2012, no.7 : 298375, 2012. DOI: $10.1155 / 2012 / 298375$

[15] Pickering, T. G., Hall, J. E., Appel, L. J., Falkner, B. E., Graves, J., Hill, M. N., ... \& Roccella, E. J.
"Recommendations for blood pressure measurement in humans and experimental animals: part 1: blood pressure measurement in humans: a statement for professionals from the Subcommittee of Professional and Public Education of the American Heart Association Council on High Blood Pressure Research," Circulation., vol. 111, no.5, pp. 697-716, 2005. DOI: 10.1161/01.CIR.0000154900.76284. F6.

[16] Jubran, A. "Pulse Oximetry: Review," Critical Care, vol.19, no.1, pp.272, 2015. DOI: 10.1186/s13054-015-0984-8.

[17] Riebe, D., Ehrman, J. K., Liguori, G., Magal, M., \& American College of Sports Medicine (Eds.). "chapter 5,"in ACSM's guidelines for exercise testing and prescription, $10^{\text {th }}$ ed, Philadelphia: Wolters Kluwer, 2018, pp.125-129.

[18] Jackson, A. S. "Estimating maximum heart rate from age: Is it a linear relationship?," Medicine and science in sports and exercise, vol. 39, no. 5 , pp. 821, 2007. DOI: 10.1249/mss.0b013e318054d3ca.

[19] Schroeder, E. C., Franke, W. D., Sharp, R. L., \& Lee, D. C. "Comparative effectiveness of aerobic, resistance, and combined training on cardiovascular disease risk factors: a randomized controlled trial," PloS one, vol. 14, no. 1, pp.e0210292, 2019. DOI: 10.1371/journal.pone.0210292.

[20] de Freitas Santos, N. M., dos Santos Tavares, D. M., Dias, F. A., Diniz, M. A., \& Martins, N. P. F. "Functional capacity and quality of life of elderly people with a history of stroke,". Acta Scientiarum. Health Sciences, vol. 35, no.1, pp.49-51, 2013. DOI: 10.4025/actascihealthsci.v35i1.1046 3

[21] Katz-Leurer, M., \& Shochina, M. "The influence of autonomic impairment on aerobic exercise outcome in stroke patients," NeuroRehabilitation , vol. 22, no. 4, pp. 267-272, 2007. DOI:10.3233/NRE-2007-22403

[22] Taheri, M., Irandoost, K., Yousefi, S., \& Jamali, A."Effect of 8-week lower extremity weight-bearing exercise protocol and acute caffeine consumption on reaction time in postmenopausal women," Iranian Journal of Ageing, vol. 12, no. 1, pp.18-29, 2017. DOI: 10.21859/sija-120116

[23] Flansbjer, U. B., Lexell, J., \& Brogårdh, C. "Long-term benefits of progressive resistance training in chronic stroke: a 4-year follow-up," Journal of rehabilitation medicine, vol. 44, no. 3, pp.218-221, 2012.DOI: 10.2340/16501977-0936

[24] Hill, T. R., Gjellesvik, T. I., Moen, P. M. R., Tørhaug, T., Fimland, M. S., Helgerud, J., \& Hoff, J. "Maximal strength training enhances strength and functional performance in chronic stroke survivors," American journal of physical medicine \& rehabilitation, vol.91, no. 5, pp.393-400,2012.DOI:10.1097/PHM.0b013e31824ad5b8.

[25] Signal, N. E. "Strength training after stroke: Rationale, evidence and potential implementation barriers for physiotherapists," New Zealand Journal of Physiotherapy, vol. 42, no. 2, pp.101-107, 2014.

[26] Veldema, J., \& Jansen, P. "Resistance training in stroke rehabilitation: systematic review and meta-analysis," Clinical Rehabilitation, vol. 34, no. 9, pp.1173-1197, 2020. DOI: $10.1177 / 029215520932964$.

[27] Giuliano, C., Karahalios, A., Neil, C., Allen, J., \& Levinger, "The effects of resistance training on muscle strength, 
quality of life and aerobic capacity in patients with chronic heart failure-A meta-analysis," International journal of cardiology, vol. 227, pp.413-423, 2017. DOI: 10.1016/j.ijcard.2016.11.023.

[28] Pedersen, M. T., Vorup, J., Nistrup, A., Wikman, J. M., Alstrøm, J. M., Melcher, P. S., ... \& Bangsbo, J."Effect of team sports and resistance training on physical function, quality of life, and motivation in older adults,"
Scandinavian journal of medicine \& science in sports, vol. 27, no.8, pp. 852-864, 2017. DOI: 10.1111/sms.12823.

[29] Barrett, C., \& Smerdely, P. "A comparison of community-based resistance exercise and flexibility exercise for seniors," Australian Journal of Physiotherapy, vol. 48, no. 3, pp.215-219, 2002. DOI: $10.1016 / \mathrm{s} 0004-9514(14) 60226$. 